\title{
Evaluación y tratamiento polimodal del dolor musculo esquelético y abdominal en equinos
}

\section{Evaluation and polimodal treatment of pain skeletic and abdominal muscle in equines}

\section{Avaliação e tratamento polimodal da dor muscular esquelética e abdominal}

\section{em equinos}

\author{
Reyes Cardona Daniela ${ }^{1}$ y Jaramillo Hernández Dumar Alexander ${ }^{2}$ \\ ${ }^{1}$ Médico Veterinario y Zootecnista, Universidad de los Llanos y \\ ${ }^{2} \mathrm{MVZ}$, Esp. MSc. $\mathrm{PhD}_{(\mathrm{c})}$, Docente Universidad de los Llanos \\ daniela.reyes@unillanos.edu.co
}

Recibido 24 de octubre 2019, Aceptado 12 de marzo 2020

\section{RESUMEN}

El dolor es uno de los aspectos más importantes que sugieren el estado de bienestar en los animales. Debido a que no podemos evaluar directamente los estados emocionales en ellos, la evaluación del bienestar se basa en factores fisiológicos e indicadores de comportamiento. Los veterinarios confían en la interpretación de la conducta de sus pacientes con el fin de guiar el tratamiento médico más adecuado, sin embargo, la evaluación clínica del dolor en animales es un procedimiento subjetivo dominado por el sesgo personal, la percepción y la fisiología. En los últimos años las investigaciones se han centrado en la creación de escalas de dolor (escala visual análoga, escala descriptiva simple, escala de calificación numérica, escala de dolor compuesta y escalas de gestos) con el fin de facilitar al clínico la determinación del grado de dolor que experimenta el paciente. Los enfoques farmacológicos para el tratamiento del dolor en equinos deben ser basados y seleccionados para fines específicos (inflamación, lesión nerviosa, sensibilización periférica frente a central) y en función de la gravedad y el tipo de dolor (somático frente a visceral; leve o grave), sin embargo la mayoría de los medicamentos utilizados para tratar el dolor en equinos se dividen en 4 categorías amplias: medicamentos antiinflamatorios (AINE), esteroides y no esteroides, opioides, y 
agonistas $\alpha 2$, a pesar de las propiedades analgésicas de estos fármacos, existen reportes de diversos efectos adversos asociados a su utilización. La analgesia polimodal es una alternativa, si bien no contempla nuevos grupos farmacológicos, propone el uso simultáneo de varios medicamentos que, por poseer diferentes mecanismos de acción, logran mejorar el efecto analgésico final, disminuyendo los riesgos de presentación de efectos adversos.

Palabras clave: Dolor, escalas de dolor, equinos, analgesia polimodal.

\begin{abstract}
Pain is one of the most important aspects that suggest a welfare state in animals. Because we cannot directly access to emotional states in them, the assessment of well-being is based on physiological factors and behavioral indicators. Veterinarians rely on the interpretation of the behavior of their patients in order to guide the most appropriate medical treatment; however, the clinical evaluation of pain in animals is a subjective procedure dominated by personal bias, perception and physiology. In recent years, research has focused on the creation of pain scales (visual analogue scale, simple descriptive scale, numerical rating scale, time budget analysis, composite pain scale and grimace scales) in order to facilitate to the clinician the determination of the degree of pain experienced by the patient. Pharmacological approaches for the treatment of pain in horses should be based and selected for specific purposes (inflammation, nerve injury), (peripheral versus central sensitization), and depending on the severity and type of pain (somatic versus visceral; mild or severe), however most of the drugs used to treat pain in horses are divided into 4 broad categories: anti-inflammatory drugs (NSAIDs), steroids and nonsteroids, opioids, and $\alpha 2$ agonists, despite the analgesic properties of These drugs, there are reports of various adverse effects associated with their use. The polymodal analgesia is an alternative, although it does not contemplate new pharmacological groups, it proposes the simultaneous use of several medications that by having different mechanisms of action, manage to improve the final analgesic effect, diminishing the risks of presentation of adverse effects.
\end{abstract}


Keywords: Pain, pain scales, equine, polymodal analgesia.

\section{RESUMO}

A dor é um dos aspectos mais importantes que sugerem o estado de bem-estar dos animais. Como não podemos avaliar diretamente os estados emocionais neles, a avaliação do bem-estar é baseada em fatores fisiológicos e indicadores comportamentais. Os médicos veterinários confiam na interpretação do comportamento de seus pacientes para orientar o tratamento médico mais adequado, no entanto, a avaliação clínica da dor em animais é um procedimento subjetivo dominado pelo viés pessoal, percepção e fisiologia. Nos últimos anos, a pesquisa concentrou-se na criação de escalas de dor (escala visual analógica, escala descritiva simples, escala numérica, escala composta de dor e escala gestual), a fim de facilitar ao clínico determinar o grau de dor que o paciente experimenta. As abordagens farmacológicas para o tratamento da dor em equinos devem ser baseadas e selecionadas para fins específicos (inflamação, lesão nervosa, sensibilização periférica versus central) e dependendo da gravidade e do tipo de dor (somática vs. visceral; leve ou grave ), no entanto, a maioria dos medicamentos usados no tratamento da dor em equídeos se enquadra em quatro grandes categorias: anti-inflamatórios (AINEs), esteróides e não esteróides, opióides e a2-agonistas, apesar das propriedades analgésicas desses medicamentos, Há relatos de vários efeitos adversos associados ao seu uso. A analgesia polimodal é uma alternativa, embora não contemple novos grupos farmacológicos, propõe o uso simultâneo de vários fármacos que, por possuírem diferentes mecanismos de ação, conseguem melhorar o efeito analgésico final, reduzindo os riscos de apresentar efeitos adversos.

Palavras-chave: dor, escalas de dor, equinos, analgesia polmodal.

\section{INTRODUCCIÓN}

El dolor es una experiencia sensorial y emocional aversiva que representa la conciencia del animal hacia un daño o amenaza a la integridad de sus tejidos (Molony y Kent, 1997). La incomodidad o el dolor son las formas mediante las cuales 
el organismo se protege, al inhibir otras actividades se promueve la recuperación ya que se evita provocar un daño adicional a los tejidos (Bateson, 1991). Aparte de las diferencias de especies, la expresión del dolor también es dependiente del tipo y origen del dolor. El dolor somático y visceral, por ejemplo, son fenómenos que se manifiestan de manera diferente y deben tratarse de manera distinta (Robertson, 2002). Por su parte el dolor visceral hace referencia al dolor que se origina en cualquier órgano interno y con frecuencia se subdivide en los órganos contenidos dentro de cada cavidad corporal principal: tórax, abdomen y pelvis (Mair et al., 2002), a su vez el dolor somático es aquel dolor debido a lesión o enfermedad de estructuras inervadas por el sistema somático, es decir: huesos, músculos y tendones (Salazar, 2012).

Debido a que no podemos evaluar directamente los estados afectivos de los animales, la evaluación del bienestar se basa en factores fisiológicos e indicadores de comportamiento (Harding et al., 2004; Mendl et al., 2010). Sin embargo, como el dolor es una experiencia multidimensional compleja no hay un solo parámetro que específicamente indique la presencia de dolor (Büttner y Finke, 2000). En la práctica veterinaria, el diagnóstico adecuado y el tratamiento del dolor dependen de la experiencia y conocimiento del clínico en reconocer e identificar el dolor en animales no verbales. Para facilitar la identificación del dolor se han desarrollado diferentes métodos, como las escalas de dolor para equinos, la mayoría de estas se basan en comportamientos y/o posturas de cabeza, cuello, orejas, cola, y a menudo la evaluación está asociada con parámetros fisiológicos (Ashley et al., 2005). Estas escalas permiten determinar el grado de dolor que experimenta el animal mejorando así la regulación analgésica y, en consecuencia, el bienestar de estos pacientes.

En la actualidad pese a las investigaciones existe un número limitado de analgésicos disponibles para tratar el dolor severo en equinos, los analgésicos más comúnmente usados incluyen los $\alpha 2$ - adrenérgicos, agonistas antiinflamatorios no esteroideos (AINEs) y opioides (Robertson y Sánchez, 2010). Sin embargo, existen diversas desventajas en la utilización de estos fármacos en terapias prolongadas, los $\alpha 2$ - adrenérgicos disminuyen la motilidad gastrointestinal, producen hipertensión 
y bradicardia seguidas de hipotensión (Elfenbein et al., 2009). Los AINEs tienen propiedades analgésicas y antiinflamatorias viscerales bien documentadas, pero sus efectos adversos incluyen: ulceración gástrica y colónica, deterioro de la restitución del epitelio yeyunal, lesiones isquémicas y necrosis tubular renal (Kalpravidh et al., 1984; MacAllister et al., 1993). Por otra parte, la administración de opioides ha resultado en efectos adversos, predominantemente relacionados con excitación y disminución de la motilidad gastrointestinal (Bennett y Steffey, 2002). La terapia polimodal es una opción alternativa para tratar el dolor, en esta se disminuyen la cantidad de efectos adversos, ya que se combinan diferentes medicamentos, reduciendo las dosis necesarias y potenciando el efecto de cada fármaco (Matthews y Carroll, 2007).

Los equinos a diferencia de los seres humanos no poseen actualmente un "estándar de oro" para la evaluación del dolor (Ashley et al., 2005). A pesar que los equinos demuestran una serie de señales conductuales de dolor, la estimación del dolor en ellos se ha considerado un desafío para los clínicos (Love et al., 2009). La raza y la variación interindividual son elementos que también deben ser considerados en la expresión del dolor (Wagner, 2010). En la actualidad el uso de sistemas de puntuación o escalas definidas y validadas ayudan al veterinario a mejorar el reconocimiento y evaluar el tratamiento de condiciones dolorosas en equinos (Dutton et al., 2009).

Las alternativas terapéuticas que existen para el tratamiento del dolor actualmente siguen siendo limitadas, a pesar de que la farmacología y la medicina veterinaria continúan avanzando la oferta de analgésicos eficaces y seguros para animales sigue siendo escasa y la utilización de los mismos se ha centrado en unos pocos fármacos principalmente pertenecientes al grupo de los antiinflamatorios no esteroidales (AINEs) (Matthews y Carroll, 2007). La analgesia polimodal es una alternativa, si bien no contempla nuevos grupos farmacológicos, propone el uso simultáneo de varios medicamentos que, por poseer diferentes mecanismos de acción, logran mejorar el efecto analgésico final, disminuyendo los riesgos de presentación de efectos adversos (Matthews y Carroll, 2007). Es de vital importancia 
para el clínico conocer los mecanismos fisiopatológicos relacionados con el dolor, y los métodos de abordaje de la clasificación del dolor en la clínica equina, esto con el fin de que el veterinario realice una correcta selección de una terapia analgésica balanceada en equinos, en la que no solo se deben tener en cuenta la eficacia de los fármacos a administrar sino también el riesgo de presentación de efectos adversos.

Este trabajo busca reunir información necesaria para poder oriental al veterinario sobre los aspectos relacionados con los mecanismos fisiológicos del dolor, sus métodos de identificación y las alternativas terapéuticas existentes con el fin de garantizar el correcto manejo de los estados dolorosos que afectan a los equinos.

\section{FISIOPATOLOGÍA DEL DOLOR}

Nocicepción es un término usado para describir el proceso neuronal para la transducción (detección), transmisión, modulación, proyección y procesamiento central de un estímulo actual o potencial de daño tisular (Muir y Woolf, 2001). La nocicepción inicia en la periferia y es dependiente de la sensibilidad y actividad de los nociceptores, neuronas nociceptivas y sistema nervioso central (medula espinal y cerebro). Bajo circunstancias normales el estímulo nocivo (mecánico, térmico, químico y/o eléctrico) activa una variedad de umbrales y nociceptores polimodales ubicados en todo el cuerpo. Las neuronas nociceptivas expresan canales de potencial de receptor transitorio (TRP) los cuales se someten a una función y están altamente implicados en la detección inmediata de estímulos nocivos (White et al., 2010).

La sensibilización central resulta de los efectos acumulativos de la repetición de la entrada nociceptiva sostenida en el cuerno dorsal de la médula espinal y la posterior liberación de glutamato y neuropéptidos (sustancia $P$ y neurocinina $A$ ) que activan receptores de N-Metil-D-Aspartato (NMDA) y taquiquininas, dando como resultado una "finalización" gradual de las vías nerviosas centrales (Muir y Woolf, 2001; Blackshaw y Gabhart, 2002). El dolor severo o prolongado (crónico) puede ocasionar sensibilización central, que a menudo se inicia por sensibilización 
periférica y se caracteriza con frecuencia por hiperalgesia, alodinia e hipersensibilidad secundaria (dolor fuera del área lesionada) (Bueno et al., 1997) por otra parte el dolor visceral (dolor intestinal, hepático, esplénico, o renal) a diferencia del dolor somático, es transmitido por fibras nerviosas parasimpáticas (principalmente vágales) y simpáticas a través de fibras del nervio esplácnico aferente (Price et al., 2003; Gleerup y Lindergaard, 2016).

\section{TIPOS DE DOLOR}

La evaluación de una lesión aguda o crónica debe incluir la determinación del dolor relacionado con daño tisular, para ello es importante que el clínico conozca la diferencia en los tipos de dolor (somático, visceral) existentes que pueden padecer los equinos, esto con el fin de facilitar el desarrollo de un adecuado tratamiento y rehabilitación pertinente para cada paciente.

El dolor visceral se puede definir como el dolor que se origina en cualquier órgano interno y a menudo se divide para incluir los órganos contenidos dentro de cada cavidad corporal principal, que son: tórax, abdomen y pelvis. Mair et al., (2002) afirman que hay aproximadamente 100 condiciones que resultan en dolor abdominal en el equino, pero las fuentes más comunes son el intestino delgado y grueso, es por esto que este tipo de dolor ha recibido mayor investigación y atención clínica en los últimos años. La causa del dolor visceral puede ser orgánica (estructural identificable cambio en un órgano) o disfuncional (un cambio anormal en la función del órgano sin cambios patológicos identificables) (Giamberardino, 2005).

El dolor visceral es un desafío para el clínico porque puede ser difícil hacer un diagnóstico definitivo; idealmente, el tratamiento está dirigido a abordar la patología subyacente, pero a menudo es complejo identificar con exactitud el órgano afectado dentro de la cavidad (Hunter, 2009).

Por otra parte, el dolor somático es aquel dolor debido a lesión o enfermedad de estructuras inervadas por el sistema somático, como son huesos, músculos y tendones. La osteoartritis (OA), también conocida como osteoartrosis o enfermedad 
articular degenerativa, es el trastorno musculo esquelético crónico más importante tanto en humanos como en equinos (Leigh et al., 2001).

\section{MÉTODOS DE EVALUACIÓN DEL DOLOR EN EQUINOS}

Existen numerosos desafíos para diagnosticar el dolor en equinos entre los principales encontramos: la falta en el reconocimiento temprano o detección de signos sutiles de dolor y la posible supresión del comportamiento de dolor dado por un estímulo de amenaza potencial (Taylor et al., 2002).

Para lograr que la evaluación del dolor sea objetiva y lo más consistente posible, es imperativo: (I) determinar cuáles comportamientos y parámetros fisiológicos pueden estar asociados con dolor, (II) realizar observaciones sistematizadas de estos signos, (III) especificar el grado de dolor relacionado con un cierto tipo de comportamiento y (IV) combinar estas observaciones en un puntaje cuantitativo, útil para estimar la intensidad del dolor (Wagner, 2010). En la actualidad se han desarrollado varias escalas del dolor con el fin de realizar una detección y cuantificación temprana de la gravedad del dolor y recomendar el tratamiento más oportuno dependiendo del tipo de patología que esté afectando al paciente. Para que un sistema de puntuación de dolor funcione de manera confiable en la práctica, debe ser fácil de usar, con parámetros relevantes bien definidos que puedan ser evaluados repetidamente y rápidamente por diferentes observadores con resultados consistentes (Raekallio et al., 1997).

\section{MEDICIÓN DE PARÁMETROS FISIOLÓGICOS}

El análisis de la variabilidad de la frecuencia cardiaca (AVFC) proporciona un método para evaluar el impacto del sistema nervioso autónomo en su función cardiovascular, debido a que la entrada autonómica también está influenciada por la amígdala (emoción) y corteza (conciencia), el AVFC sirve como un indicador de efecto emocional (Lang et al., 2000). Además de la frecuencia cardiaca, la frecuencia respiratoria también puede verse afectada por el dolor, estos dos parámetros tienen la ventaja que son fácilmente medibles y cuantificables, es por esto que en la clínica diaria son indicadores claves de dolor y necesidad de 
analgesia (Price et al., 2003; Dujardin y van Loon, 2011). Sin embargo, estos parámetros solos son poco específicos para determinar la presencia y severidad de dolor, debido a que pueden ser influenciados por otros factores como: la temperatura ambiental, deshidratación, excitabilidad y enfermedad cardiovascular y/o respiratoria (Raekallio et al., 1997; Dzikiti et al., 2003).

\section{ESCALA VISUAL ANÁLOGA}

En veterinaria "la escala visual análoga o VAS por sus siglas en inglés, consiste en una línea horizontal de $10 \mathrm{~cm}$, que representa la intensidad del dolor, esta inicia desde la parte izquierda de la línea la cual hace referencia a la ausencia de dolor, hacia el lado derecho de la línea se va aumentando el grado de dolor hasta llegar al peor dolor inimaginable. En la práctica equina, diferentes observadores alcanzaran diferentes puntuaciones para el mismo paciente y situación (conocido como pobre acuerdo inter observador). Esto hace que esta escala sea inadecuada para un entorno hospitalario donde varios observadores evaluaran al mismo paciente, sin embargo, la escala VAS puede ser útil para observación del propietario sobre el mismo animal durante un curso prolongado (Hielm-Bjorkman et al. 2011).

\section{ESCALA NUMÉRICA}

La escala numérica (EN) consiste en una línea horizontal con etiquetas numéricas preestablecidas de 0 a 10 . Nuevamente, cero designa sin dolor y 10 el peor dolor imaginable. Los observadores deben encerrar en un círculo el número más cercano a la cantidad percibida del dolor que el animal está experimentando (Ashley et al., 2005). Se considera que la escala numérica es menos sensible para identificar pequeños cambios en el dolor comparado con el VAS, ya que solo preestablece las entradas de números enteros (Viñuela-Fernández et al., 2011).

\section{ESCALA DESCRIPTIVA SIMPLE (EDS)}

La EDS consiste en grados de dolor a los cuales se les asigna un número de índice para permitir que los datos se manejen estadísticamente, p.ej. 0, ninguno; 1 , dolor leve; 2 , dolor moderado; 3 , dolor severo. Se espera que las escalas descriptivas 
simples funcionen mejor que la escala visual análoga o la escala numérica en manos de observadores menos experimentados (dado que se proporcionan clases predefinidas), este no es siempre el caso (Kester, 1991). Ejemplos del uso de las EDS en la práctica clínica incluye los sistemas de clasificación de cojeras, como el de la Asociación estadunidense de Practicantes Equinos (AAEP), o la clasificación de Obel para laminitis (Price et al., 2003).

\section{ESCALAS DEL DOLOR COMPUESTAS}

Dado que el dolor es un fenómeno subjetivo, multidimensional y complejo que evoca respuestas emocionales, conductuales y fisiológicas su evaluación requiere identificar parámetros puntuales relacionados con el dolor con el fin de determinar y cuantificar mejor los estados de dolor que aquejan a los equinos (Dobromylskyj et al., 2000). Para lograr este objetivo se construyen las escalas de dolor compuestas (EDC), donde múltiples variables (conductuales fisiológicas o ambas) se clasifican individualmente utilizando clases bien definidas por medio de escalas descriptivas simples, cada una de estas va a describir un comportamiento específico o un parámetro fisiológico en cuatro o cinco grados claramente definidos. Cada EDS es evaluada individualmente y posteriormente todos los puntajes son sumados para obtener un puntaje de dolor final" (Sutton et al., 2013).

\section{TEST DE ANALGESIA}

La validación de los comportamientos relacionados con el dolor equino se puede lograr de manera efectiva estudiando la respuesta a la analgesia, aunque la mayoría de los estudios en esta el área están dirigidos a las pruebas de eficacia analgésica (Gleerup y Lindegaard, 2014). En estos caballos, se realiza tratamiento analgésico durante el entrenamiento con el fin de evaluar si el bajo rendimiento o problemas de conducción están relacionados con dolor. Si el comportamiento se reduce o elimina después del tratamiento (y específicamente si se repite después de que se haya disipado el efecto del analgésico), entonces es probable que el problema se deba a una afección dolorosa no diagnosticada (Pozzi et al., 2006). 


\section{APLICACIÓN DE HERRAMIENTAS DE EVALUACIÓN SISTEMÁTICA A ESTADOS DE DOLOR ESPECÍFICOS EN EQUINOS}

\section{Laminitis}

La laminitis aguda se considera una de las condiciones más dolorosas que un caballo puede experimentar (Wagner, 2010; Dujardin y van Loon, 2011). La escala de dolor de laminitis desarrollada por Nils Obel es una EDS que clasifica la gravedad de la cojera debido a la laminitis del grado de I al IV (Obel, 1948; Wagner, 2010); (Tabla 1).

Tabla 1. Escala de dolor de laminítis

\begin{tabular}{cl}
\hline Grado I & $\begin{array}{l}\text { El animal se encuentra en posición de pie y levanta } \\
\text { incansablemente los miembros con intervalos sólo de unos pocos } \\
\text { segundos. Al caminar no muestra cojera pero al trote se observa } \\
\text { leve disfunción. }\end{array}$ \\
Gamina aún sin cojera, pero al trote muestra clara claudicación de \\
apoyo bilateral, adelantando los miembros al trote, levanta los \\
miembros sin dificultad.
\end{tabular}

Fuente: Escala de dolor de laminítis (Obel, 1948)

Viñuela-Fernández et al. (2011) evaluaron la confiabilidad de tres sistemas subjetivos de puntuación para laminitis: EVA (escala visual análoga), EDS (escala descriptiva simple) y la puntuación de Obel modificada adaptada por Owens et al. (1995) y el "sistema de calificación clínica" desarrollado por Taylor et al., (2002), la confiabilidad general de los tres sistemas de puntuación fue alta, con confiabilidad entre observadores consistentemente alta (Viñuela-Fernández et al., 2011).

\section{Sinovitis}

Una causa importante de cojeras en los equinos es la sinovitis aguda. Estudios experimentales utilizando inyecciones intraarticulares (IA) de lipopolisacárido (LPS) o anfotericina B para inducir sinovitis aguda o crónica, respectivamente, Se han 
utilizado para validar herramientas de puntuación de dolor y monitorear la eficacia de la intervención analgésica (Bussieres et al., 2008; de Grauw et al., 2009a, 2014; Lindegaard et al., 2010; van Loon et al., 2010). Usando el modelo de anfotericina B, se desarrolló una escala de dolor compuesta para ortopedia validada por Bussières et al., (2008). Esta EDC incluye varias variables de comportamiento interactivas (como la respuesta a la palpación del área afectada), así como la observación (por ejemplo, la postura) y variables fisiológicas (Tabla 2). De las variables incluidas, la respuesta a la palpación del área afectada y la postura fueron los indicadores más sensibles y específicos de dolor asociados a sinovitis severa.

Tabla 2. Escala de dolor compuesta de calificación numérica multifactorial

\begin{tabular}{|c|c|c|}
\hline Datos fisiológicos & Criterio & Puntaje /12 \\
\hline \multirow{4}{*}{$\begin{array}{c}\text { Frecuencia } \\
\text { cardiaca }\end{array}$} & Normal comparado con el valor inicial ( $<10 \%$ aumento) & 0 \\
\hline & $11-30 \%$ aumento & 1 \\
\hline & $31-50 \%$ aumento & 2 \\
\hline & $>50 \%$ aumento & 3 \\
\hline \multirow{4}{*}{$\begin{array}{l}\text { Frecuencia } \\
\text { respiratoria }\end{array}$} & Normal comparado con el valor inicial ( $<10 \%$ aumento) & 0 \\
\hline & $11-30 \%$ aumento & 1 \\
\hline & $31-50 \%$ aumento & 2 \\
\hline & $>50 \%$ aumento & 3 \\
\hline \multirow{4}{*}{ Sonidos digestivos } & Motilidad normal & 0 \\
\hline & Disminución de la motilidad & 1 \\
\hline & Amotil & 2 \\
\hline & Hipermotil & 3 \\
\hline \multirow{4}{*}{ Temperatura rectal } & Normal comparado con el valor inicial (variación $<0,5^{\circ} \mathrm{C}$ ) & 0 \\
\hline & Variación $<1^{\circ} \mathrm{C}$ & 1 \\
\hline & Variación mayor $1,5^{\circ} \mathrm{C}$ & 2 \\
\hline & Variación $2^{\circ} \mathrm{C}$ o mas & 3 \\
\hline \multirow{5}{*}{$\begin{array}{c}\text { Respuesta al } \\
\text { tratamiento } \\
\text { Comportamiento de } \\
\text { interacción }\end{array}$} & & Puntaje/6 \\
\hline & Pone atención a las personas & 0 \\
\hline & Respuesta exagerada a estímulos auditivos & 1 \\
\hline & Respuesta excesiva-agresiva a estímulos auditivos & 2 \\
\hline & Stupor, postración no respuesta a estímulos auditivos & 3 \\
\hline \multirow{4}{*}{$\begin{array}{c}\text { Respuesta a ala } \\
\text { palpación del área } \\
\text { afectada }\end{array}$} & No haya reacción a la palpación & 0 \\
\hline & Mediana reacción a la palpación & 1 \\
\hline & Se resiste a la palpación & 2 \\
\hline & Violenta reacción a la palpación & 3 \\
\hline
\end{tabular}




\begin{tabular}{|c|c|c|}
\hline \multirow{5}{*}{$\begin{array}{c}\text { Comportamiento } \\
\text { Se patea el } \\
\text { abdomen }\end{array}$} & & Puntaje/21 \\
\hline & $\begin{array}{l}\text { De pie en silencio sin patear } \\
\text { Din }\end{array}$ & 0 \\
\hline & Ocasionalmente patea el abdomen $(1-2 \times / 5 \mathrm{~min})$ & 1 \\
\hline & frecuentemente patea el abdomen ( $3-4$ x/ 5 min) & 2 \\
\hline & $\begin{array}{l}\text { Excesivamente patea el abdomen }(>5 \times / 5 \mathrm{~min}) \\
\text { intermitentemente intenta acostarse y rodar }\end{array}$ & 3 \\
\hline \multirow{4}{*}{$\begin{array}{l}\text { Patear-raspar el } \\
\quad \text { piso }\end{array}$} & De pie en silencio sin patear & 0 \\
\hline & Ocasionalmente patea el piso (1-2 x/ 5 min) & 1 \\
\hline & frecuentemente patea el piso ( $3-4$ x/ 5 min) & 2 \\
\hline & Excesivamente patea el piso (>5 x/ 5 min) & 3 \\
\hline \multirow{4}{*}{$\begin{array}{l}\text { Postura (peso, } \\
\text { distribución, } \\
\text { confort) }\end{array}$} & Se para normal en silencio, camina normal & 0 \\
\hline & $\begin{array}{c}\text { Ocasionalmente ladea su peso, leves tremores } \\
\text { musculares }\end{array}$ & 1 \\
\hline & No hay soporte de peso, distribución anormal de peso & 2 \\
\hline & $\begin{array}{c}\text { Postura analgésica (intentos de orinar, postración, } \\
\text { tremores musculares) }\end{array}$ & 3 \\
\hline \multirow{4}{*}{$\begin{array}{l}\text { Movimientos de la } \\
\text { cabeza }\end{array}$} & No hay evidencia de discomfort, cabeza derecha & 0 \\
\hline & $\begin{array}{l}\text { Movimientos intermitentes laterales o vertical } \\
\text { ocasionalmente observando el flanco (1-2 x/5 min) }\end{array}$ & 1 \\
\hline & $\begin{array}{l}\text { Movimientos rápidos e intermitentes laterales y verticales } \\
\qquad(3-4 \times / 5 \mathrm{~min})\end{array}$ & 2 \\
\hline & $\begin{array}{l}\text { Movimientos de cabeza continuos, observación excesiva } \\
\text { del flanco }(>5 x / 5 \mathrm{~min})\end{array}$ & 3 \\
\hline \multirow{5}{*}{ Apetito } & Come normalmente & 0 \\
\hline & Vacila para comer & 1 \\
\hline & $\begin{array}{l}\text { Muestra un poco de interés, come poco, o introduce en } \\
\text { alimento a la boca pero no mastica o traga }\end{array}$ & 2 \\
\hline & No muestra interés, no come & 3 \\
\hline & Total & 39 \\
\hline
\end{tabular}

Fuente: Bussieres et al. (2008).

Se debe elegir un valor de 0-3 para cada comportamiento, se hace referencia a 0: normal, 3: lejos de lo normal, posteriormente se realiza una tabulación de los resultados indicando 1-10 dolor bajo; 10-20 dolor moderado; >20 dolor severo.

\section{Castración}

Aunque se considera un procedimiento rutina, la castración es una cirugía invasiva con el potencial a desarrollar considerables complicaciones postquirúrgicas y dolor postoperatorio. Se han realizado varios estudios con diferentes escalas para 
determinar el grado de dolor y efectividad de la analgesia en procedimientos de castración, la escala visual análoga y la escala descriptiva simple, se han utilizado para evaluar la eficacia de la buprenorfina y butorfanol para el dolor posterior a la castración en ponies (Love et al., 2013), sin embargo, se ha utilizado la castración quirúrgica para desarrollar una escala de expresión facial basada en seis parámetros (Tabla 3).

Tabla 3. Escala de gestos en equinos

\begin{tabular}{|c|c|c|}
\hline Acción facial & Descripción & Puntaje \\
\hline \multirow{3}{*}{$\begin{array}{l}\text { Orejas rígidamente } \\
\text { hacia atrás }\end{array}$} & $\begin{array}{l}\text { Las orejas se sostienen rígidamente y se giran } \\
\text { hacia atrás. }\end{array}$ & 0 \\
\hline & Moderadamente presente & 1 \\
\hline & Obviamente presente & 2 \\
\hline \multirow{3}{*}{ Ajuste orbital } & $\begin{array}{l}\text { El párpado está parcial o completamente cerrado. } \\
\text { No presente }\end{array}$ & 0 \\
\hline & Moderadamente presente & 1 \\
\hline & Obviamente presente & 2 \\
\hline \multirow{3}{*}{$\begin{array}{l}\text { Tensión en el área } \\
\text { superior del ojo }\end{array}$} & No presente & 0 \\
\hline & Moderadamente presente & 1 \\
\hline & Obviamente presente & 2 \\
\hline \multirow{3}{*}{$\begin{array}{l}\text { Tensión de los } \\
\text { músculos } \\
\text { masticatorios }\end{array}$} & $\begin{array}{l}\text { La tensión de los músculos masticatorios es } \\
\text { claramente visible como un aumento de la tensión } \\
\text { por encima de la boca si al masticar. No presente }\end{array}$ & 0 \\
\hline & Moderadamente presente & 1 \\
\hline & Obviamente presente & 2 \\
\hline \multirow{3}{*}{$\begin{array}{l}\text { Boca tensa y } \\
\text { barbilla } \\
\text { pronunciada }\end{array}$} & $\begin{array}{l}\text { La boca estirada es claramente visible cuando el } \\
\text { labio superior. No presente }\end{array}$ & 0 \\
\hline & Moderadamente presente & 1 \\
\hline & Obviamente presente & 2 \\
\hline \multirow{4}{*}{$\begin{array}{l}\text { Fosas nasales } \\
\text { tensas y } \\
\text { aplanamiento del } \\
\text { perfil }\end{array}$} & $\begin{array}{c}\text { Las fosas nasales se ven tensas y ligeramente } \\
\text { dilatadas, el perfil de la nariz se aplana y los labios } \\
\text { se alargan }\end{array}$ & \\
\hline & No presente & 0 \\
\hline & Moderadamente presente & 1 \\
\hline & Obviamente presente & 2 \\
\hline $\begin{array}{l}\text { Puntaje total de } \\
\text { dolor }\end{array}$ & & 12 \\
\hline
\end{tabular}

Fuente: Dalla et al., (2014). 
Para la aplicación de esta tabla se debe llevar a cabo la elección de un valor de 020 : indicando no presente 1: moderadamente presente y 2 : obviamente presente, posteriormente se suman los resultados obtenidos para indicar: 1-4: dolor leve; 4-7 dolor moderado > 7: dolor severo.

\section{Cólico agudo}

El cólico es una de las causas más importantes de morbilidad y mortalidad en equinos (Freeman y Curtis, 2015). El cólico agudo se asocia con la expresión abierta de dolor en equinos y la intensidad del comportamiento asociado al dolor puede guiar la decisión quirúrgica (Ashley et al., 2005). Sutton et al., (2013) desarrollaron y validaron dos escalas de dolor basadas en el comportamiento para equinos con cólicos agudos (EAAPS-1 y -2 ); estas escalas multivariables se basan principalmente en componentes de comportamiento observacional (Tablas 4 y 5 ) sin incluir parámetros fisiológicos; no son escalas compuestas de dolor, ya que no se agregan sub puntuaciones para obtener una puntuación general de dolor.

Tabla 4. Escala de dolor abdominal agudo en equinos - versión I (EAAPS-1)

\begin{tabular}{ccc}
\hline Tipo & Comportamiento & Puntaje \\
\hline & Depresión & 1 \\
& Observación del flanco & 2 \\
Distribución del peso & 2 \\
intranquilidad & 3 \\
Moderado & Pateo del abdomen & 3 \\
& Raspado del suelo & 3 \\
& Estiramiento & 3 \\
& Recumbencia esternal & 3 \\
& Intentos de acostarse & 3 \\
& Recumbencia lateral & 4 \\
Severo & Rolling & 4 \\
& Colapso & 5 \\
\hline
\end{tabular}

Fuente: Sutton et al., (2013)

Para determinar la severidad del dolor que experimenta el animal elija el comportamiento más severo que manifiesta el equino, y el puntaje para ese 
comportamiento estará en la escala de dolor, interpretándose así: 1-2: dolor leve; 24: dolor moderado 5: dolor severo.

Tabla 5. Escala de dolor abdominal agudo en equinos - versión 2 (EAAPS-2)

\begin{tabular}{|c|c|c|c|c|c|c|}
\hline \multirow{2}{*}{ Dolor } & \multirow{2}{*}{ Comportamiento } & \multicolumn{5}{|c|}{ Dolor } \\
\hline & & Moderado & & & vero & \\
\hline \multirow{7}{*}{ Moderado } & Depresión & 1 & & & & \\
\hline & Observación del flanco a & 1 & 2 & & & \\
\hline & Distribución del peso a & 1 & 2 & & & \\
\hline & Raspado del suelo a & & 2 & 3 & & \\
\hline & Estiramiento $^{\mathrm{a}}$ & & 2 & 3 & & \\
\hline & $\begin{array}{c}\text { Pateo del abdomen }{ }^{a} \\
\text { Intranquilidad }{ }^{b} \\
\text { Recumbencia esternal }{ }^{c}\end{array}$ & & $\begin{array}{l}2 \\
2\end{array}$ & $\begin{array}{l}3 \\
3 \\
3\end{array}$ & 4 & \\
\hline & $\begin{array}{l}\text { Intentos de acostarse } \\
\text { Recumbencia lateral } \\
\text { Rodar }\end{array}$ & & & & $\begin{array}{l}4 \\
4\end{array}$ & 5 \\
\hline Severo & Colapso & & & & & 5 \\
\hline
\end{tabular}

a El puntaje más bajo aplica si el comportamiento es ocasionalmente o raramente observado, el puntaje más alto se utiliza cuando el comportamiento es realizado frecuentemente o si se realiza de manera exagerada. ${ }^{b}$ El puntaje más bajo aplica a un caballo que camina en círculos en la pesebrera, pivotea alrededor, o se desplaza sin razón aparente, pero solo ocasionalmente, y el puntaje más alto aplica a los equinos que se desplazan continuamente, o lo realizan de una manera violenta. ${ }^{\circ}$ El puntaje más bajo aplica a un equino que esta alerta, con la cabeza levantada y el puntaje más alto se da cuando la cabeza del animal reposa en el suelo o un lado del equino.

Fuente: Sutton et al., (2013)

Para determinar la severidad del dolor que experimenta el animal elija el comportamiento más severo que manifiesta el equino, luego elija alguno de los dos puntajes para ese comportamiento en específico basándose en las descripciones en la parte inferior de la tabla.

\section{Cirugía abdominal}

La cirugía de emergencia para cólico es un procedimiento común en la práctica equina, y el dolor asociado con coeliotomia, puede ser substancial. Múltiples estudios han tendido a desarrollar escalas para determinar o cuantificar el dolor 
después de una cirugía abdominal lo cual puede ser bastante útil para tomar decisiones clínicas y determinar un pronóstico.

La escala numérica desarrollada por Pritchett et al., (2003) usa indicadores fisiológicos y de comportamiento para dolor postoperatorio, con el fin de determinar la eficacia de la intervención analgésica, estos mismos autores encontraron que los caballos postquirúrgicos pasan mayor tiempo desarrollando comportamientos de dolor y menos tiempo en procesos de locomoción voluntaria. Tal escala asigna un puntaje numérico a cada categoría de comportamiento basado en la descripción del comportamiento (Tabla 6).

Tabla 6. Escala numérica de comportamiento

\begin{tabular}{|c|c|c|c|c|}
\hline $\begin{array}{l}\text { Categoría de } \\
\text { comportamiento }\end{array}$ & 1 & 2 & 3 & 4 \\
\hline $\begin{array}{l}\text { Comportamiento } \\
\text { rudo a,b }\end{array}$ & Ninguno & & Ocasionalmente & Continuamente \\
\hline $\begin{array}{l}\text { Posición de } \\
\text { cabeza b }\end{array}$ & Encima de la cruz & & Al nivel de la cruz & $\begin{array}{c}\text { Por debajo de la } \\
\text { cruz }\end{array}$ \\
\hline $\begin{array}{c}\text { Posición de } \\
\text { orejas }^{b}\end{array}$ & $\begin{array}{l}\text { Hacia adelante, } \\
\text { movimiento } \\
\text { frecuente }\end{array}$ & & $\begin{array}{c}\text { Ligeramente } \\
\text { hacia atrás, poco } \\
\text { movimiento }\end{array}$ & \\
\hline $\begin{array}{l}\text { Localización en } \\
\text { la pesebrera }{ }^{b}\end{array}$ & $\begin{array}{l}\text { En la puerta } \\
\text { observando el } \\
\text { ambiente }\end{array}$ & $\begin{array}{l}\text { Se para en la } \\
\text { mitad, } \\
\text { mirando hacia } \\
\text { la puerta }\end{array}$ & $\begin{array}{l}\text { Se para en la } \\
\text { mitad, mira hacia } \\
\text { un lado de la } \\
\text { pesebrera }\end{array}$ & $\begin{array}{l}\text { Se para en la } \\
\text { mitad, da la } \\
\text { espalda a la } \\
\text { pesebrera }\end{array}$ \\
\hline $\begin{array}{l}\text { Locomoción } \\
\text { espontánea }\end{array}$ & $\begin{array}{l}\text { Se mueve } \\
\text { libremente }\end{array}$ & $\begin{array}{c}\text { Pasos } \\
\text { ocasionales }\end{array}$ & & $\begin{array}{l}\text { No hay } \\
\text { movimiento }\end{array}$ \\
\hline $\begin{array}{l}\text { Respuesta a la } \\
\text { apertura de la } \\
\text { puerta }^{c}\end{array}$ & $\begin{array}{c}\text { Se va hacia la } \\
\text { puerta }\end{array}$ & Mira la puerta & & No hay respuesta \\
\hline $\begin{array}{l}\text { Respuesta al } \\
\text { acercarse }^{c}\end{array}$ & $\begin{array}{l}\text { Se desplaza } \\
\text { hacia el } \\
\text { observador, } \\
\text { orejas hacia } \\
\text { adelante }\end{array}$ & $\begin{array}{l}\text { Mira al } \\
\text { observador } \\
\text { orejas hacia } \\
\text { atrás }\end{array}$ & $\begin{array}{l}\text { Se aleja del } \\
\text { observador }\end{array}$ & $\begin{array}{c}\text { No se mueve } \\
\text { orejas hacia atrás }\end{array}$ \\
\hline $\begin{array}{l}\text { Levantamiento } \\
\text { de patas }^{c}\end{array}$ & $\begin{array}{c}\text { Levanta la que se } \\
\text { le solicita }\end{array}$ & $\begin{array}{l}\text { Levanta las } \\
\text { patas después } \\
\text { de que se le } \\
\text { insiste }\end{array}$ & & $\begin{array}{l}\text { Extremadamente } \\
\text { sin voluntad de } \\
\text { levantar las patas }\end{array}$ \\
\hline
\end{tabular}


Respuesta al concentrado
Se desplaza a la puerta y alcanza el concentrado
Mira hacia la

puerta

No hay respuesta

a Comportamiento rudo incluye raspado del piso, sudoración, observación de los flancos, acostarse/levantarse repetidamente. ${ }^{b}$ puntajes combinados para dar un puntaje de postura. ${ }^{c}$ puntajes combinados para dar un puntaje de socialización

Fuente: Pritchett et al., (2003).

\section{MANEJO FARMACOLÓGICO POLIMODAL DEL DOLOR EN EQUINOS}

En la clínica equina en muchos casos no se puede realizar un diagnóstico definitivo de una patología o este puede tomar tiempo, lo cual retrasa la instauración terapéutica. Se ha argumentado que el manejo del dolor debe ser retenido hasta que la causa del dolor ha sido identificada porque enmascararlo confundirá el diagnóstico. Ahora bien, en la actualidad en cuanto más tiempo se demore en tratar el dolor, mayor es el riesgo de terminar en sensibilización e hiperalgesia (Robertson et al., 2005). Actualmente existen en el mercado una gran variedad de analgésicos disponibles para tratar el dolor en equinos. Los medicamentos analgésicos más comúnmente utilizados incluyen agonistas a2 adrenérgicos, fármacos antiinflamatorios no esferoidales (AINES), opioides y anestésicos locales (Tabla 7) (Sellon et al., 2001).

El dolor intenso puede ser refractario a la terapia analgésica con un solo fármaco, llegando a requerir un tratamiento multifactorial. El enfoque del manejo polimodal del dolor es una alternativa, en este se emplea el uso de varios fármacos con diferentes mecanismos de acción (Matthews y Carroll, 2007) dentro de las diferentes vías de administración se incluye la vía oral, intramuscular, subcutánea, epidural, intraauricular, aplicación regional en troncos nerviosos identificados, y las vías transdérmica e intravenosa, en esta se puede optar por administrar el medicamento mediante infusión constante con el fin de lograr un efecto analgésico sostenido (Taylor, 2007). Se considera que el mejor efecto analgésico bajo el concepto de analgesia polimodal, se logra al asociar principalmente un solo fármaco que ejerce acción analgésica a nivel central con otros que lo hacen a nivel periférico (Goodrich y Nixon, 2006). 
Tabla 7. Clases de medicamentos analgésicos

\begin{tabular}{|c|c|c|}
\hline $\begin{array}{c}\text { Clase de } \\
\text { medicamento }\end{array}$ & Mecanismo de acción & $\begin{array}{l}\text { Evidencia de } \\
\text { la eficacia } \\
\text { clínica }\end{array}$ \\
\hline Opioides & & \\
\hline $\begin{array}{l}\text { Morphina, Meperidina, } \\
\text { Methadona, } \\
\text { Hydromorphona, } \\
\text { Fentanylo, } \\
\text { Butorphanol, } \\
\text { Buprenorpina }\end{array}$ & $\begin{array}{l}\text { La actividad agonista de los opioides } \\
\text { puede ser importante para el butorfanol }\end{array}$ & Buena \\
\hline $\begin{array}{l}\text { Agonists Alpha-2 } \\
\text { Xylazina, Detomidina, } \\
\text { Medetomidina, } \\
\text { Romifidina }\end{array}$ & Estimula los receptores alpha-2 & Buena \\
\hline $\begin{array}{c}\text { AINES } \\
\text { Aspirina, } \\
\text { Phenylbutazona, } \\
\text { Flunixin, Ketoprofeno, } \\
\text { Firocoxib, Diclofenaco }\end{array}$ & $\begin{array}{c}\text { Inhibición de la cyclooxygenasa y } \\
\text { prostaglandinas: } \\
\text { PGE2, PGI2, TXA2 }\end{array}$ & Fuerte \\
\hline $\begin{array}{l}\text { Anestésicos locales } \\
\text { Lidocaina, } \\
\text { Mepivacaina, } \\
\text { Bupivacaina, } \\
\text { Ropivacaina }\end{array}$ & Bloqueo de los canales de $\mathrm{Na}^{+}$ & Fuerte \\
\hline $\begin{array}{l}\text { Corticoides } \\
\text { Triamcinolona } \\
\text { acetonida, } \\
\text { Methylprednisolona } \\
\text { acetato, } \\
\text { Dexametasona }\end{array}$ & Inhibición de la fosfolipasa A2 & Buena \\
\hline $\begin{array}{l}\text { Otros } \\
\text { Ketamina, tiletamina, } \\
\text { Gabapentina, } \\
\text { Tramadol, Capsaicina }\end{array}$ & $\begin{array}{l}\text { Antagonista NMDA (N-metil- D } \\
\text { aspartate) } \\
\text { bloqueador de los canales de } \mathrm{Ca}^{++} \\
\text {Agonistas de los receptores } \mu \text {, } \\
\text { Desensibilización de las fibras C }\end{array}$ & $\begin{array}{l}\text { Buena } \\
\text { Pobre } \\
\text { Pobre } \\
\text { Desconocida }\end{array}$ \\
\hline
\end{tabular}

Fuente: Muir, (2010) 


\section{UTILIZACIÓN DE AINES EN ANALGESIA POLIMODAL}

Los antiinflamatorios no esteroidales (AINEs: Fenilbutazona, Flunixina meglumina, Firocoxib) producen efectos antiinflamatorios y analgésicos mediante la inhibición de la ciclooxigenasa (COX), enzima encargada de metabolizar el ácido araquidónico en prostaglandinas (Bailey y Elliot, 2007). Las prostaglandinas y los leucotrienos son factores clave en la producción de sensibilización periférica y probablemente sean importantes para aumentar la sensibilización central. Los AINEs son analgésicos eficaces para el tratamiento del dolor inflamatorio leve a moderado, mientras que, en dolores severos o dolores refractarios, su eficacia es reducida (Kalpravidh et al., 1984).

Además de esto se han reportado efectos adversos asociados con el uso de AINEs en el tratamiento del dolor, entre los que se incluyen: ulceración gástrica y colónica, deterioro del yeyuno, restitución epitelial después de una lesión isquémica y necrosis tubular renal (MacAllister et al., 1993; Cook et al., 2009; Matthews y Carroll, 2007). Sin embargo, la combinación de AINEs con otros fármacos permite potenciar el efecto de ambos medicamentos debido a los diferentes mecanismos de acción que realiza cada uno para la disminución del dolor, de esta manera se reduce la cantidad de dosis requeridas, y a su vez los efectos adversos relacionados con estos (Reyes et al., 2004).

\section{UTILIZACIÓN DE OPIOIDES EN ANALGESIA POLIMODAL}

Los opioides no se han usado ampliamente para tratar el dolor clínico en caballos en comparación con otras especies, como los humanos. Las razones para esto incluyen el margen estrecho aparente entre la analgesia y la excitación, sumado a la dificultad para demostrar una acción analgésica consistente y cuantificable (Muir, 2010). Otros efectos adversos que se reportan por el uso de opioides son: predisposición a íleo, paralitico, cólico, excitación (aumento de la actividad locomotora), agitación, desorientación, y ataxia en algunos casos, Las dosis altas o repetidas de opioides pueden dar como resultado retención urinaria. Los opioides producen la mayoría de sus efectos analgésicos clínicamente relevantes mediante 
la activación de los receptores opioides mu $(\mu)$, kappa $(\mathrm{k})$, o delta $(\delta)$ (Combie et al., 1981). Los opioides disponibles actualmente incluyen agonistas- $\mu$ completos (morfina, metadona, fentanilo), agonistas- $\mu$ parciales (buprenorfina) y agonistas- $\mathrm{K} /$ antagonistas- $\mu$ (butorfanol). La morfina es el agonista- $\mu$ prototípico con que se comparan todos los demás opioides (Combie et al., 1983; Matthews y Carroll, 2007).

La eficacia analgésica de los opioides es notoria, frente a otros grupos de analgésicos, pero su uso como terapia única en la mayoría de los equinos desencadena efectos adversos no deseados como se mencionó anteriormente, sin embargo, una alternativa para su uso es la terapia complementaria o polimodal junto con otros fármacos como: AINEs, agonistas alfa-2 y anestésicos locales (Muir, 2016). Dentro de las asociaciones más frecuentes se encuentran las realizadas entre analgésicos opioides y sedantes principalmente agonistas alfa-2 y fenotiacínicos. La combinación de opioides, especialmente morfina en asociación con agonistas alfa-2 produce profundos efectos sedantes, Este estado se denomina comúnmente "neuroleptoanalgesia", y es utilizado en animales que son refractarios a la analgesia o cuando las intervenciones permanentes son necesarias (Muir et al., 1979; Bennett y Steffey, 2002).

\section{UTILIZACIÓN DE ALFA-2 ADRENÉRGICOS EN ANALGESIA POLIMODAL}

Los agonistas Alpha-2, tales como la xylazina, clonidina, romifidina, detomidina, medetomidina, y dexmedetomidina, son potentes medicamentos analgésicos que también incluyen cambios comportamentales y psicológicos, tales como hipertensión, bradicardia, bloqueo atrio ventricular, sedación excesiva y ataxia, por estos efectos su uso puede ser limitado como analgésico de uso sistémico en algunos pacientes (Daunt y Steffey, 2008).

Entre los efectos adversos más conocidos por el uso de terapias con agonista Alpha-2 encontramos: disminución de la motilidad intestinal, vasoconstricción de la musculatura cecal, disminución del vaciamiento gástrico, lesiones en la mucosa gástrica e intestinal, aumento en la producción de orina debido a la hiperglicemia (Alexander e Irvine, 2000). Para disminuir estos efectos se ha realizado la 
combinación de agonista Alpha-2 con opioides, La combinación de opioides, especialmente morfina en asociación con agonistas alfa-2 produce profundos efectos sedantes, entre las asociaciones mayormente reportadas se encuentra Xilazina + buprenorfina, Xilazina + metadona y dentro de estas combinaciones, se ha encontrado que el mejor efecto analgésico se logra con la asociación xilazina + buprenorfina (Bennett y Steffey, 2002).

\section{UTILIZACIÓN DE ANESTÉSICOS LOCALES EN ANALGESIA POLIMODAL}

Los anestésicos locales (lidocaína, mepivacaína, bupivacaína, ropivacaína) se usan frecuentemente solos o en combinación con otros analgésicos para producir pérdida de sensación de dolor, estos pueden ser administrados tópicamente, regionalmente (local, peri neural, epidural) y sistémicamente (Doherty y Frazier, 1998).

Los anestésicos locales, principalmente las lidocaínas producen mínimos efectos cardiovasculares y respiratorios en condiciones normales, pero pueden disminuir el gasto cardíaco, la presión arterial y la frecuencia cardíaca cuando se administran por vía intravenosa, debido a la disminución en el sistema nervioso central, fuerza contráctil del miocardio y retorno venoso (Rezende et al., 2011; Harkins et al., 1995). Las diferencias significativas en el metabolismo, la eliminación y el potencial de producir toxicidad en el sistema nervioso central (desorientación, ataxia y ataques) son otros de los efectos adversos que se pueden observar entre otros anestésicos locales (Matthews y Carroll, 2007).

La vía epidural es la que se utiliza con mayor frecuencia en esta analgesia polimodal, los reportes más frecuentes en equinos corresponden a la asociación de agonistas alfa-2 con anestésicos locales (Natalini y Driessen, 2007). E conocimiento de los mecanismos fisiopatológicos, los parámetros relacionados con el dolor, y los métodos de abordaje de la clasificación del dolor en la clínica equina permiten que el veterinario realice una correcta selección de una terapia analgésica balanceada en equinos, en la que no solo se deben tener en cuenta la eficacia de los fármacos a administrar sino también el riesgo de presentación de efectos adversos y de interacción medicamentosa. 


\section{ANÁLISIS Y DISCUSIÓN}

El manejo del dolor en animales, asociado a lesión o enfermedad, es un objetivo fundamental de la medicina veterinaria (Waran et al., 2010). Sin embargo, el manejo de dicho dolor es dependiente de la calidad de la evaluación del dolor que experimenta el paciente. A pesar que la medicina equina y la cirugía han avanzado significativamente durante las últimas décadas, dando como resultado procedimientos médicos y quirúrgicos más avanzados, continúa siendo aún un desafío clínico identificar y tratar correctamente los estados de dolor que afectan a los equinos (Bisgaard et al., 2001; Sellon et al., 2004). A pesar de que se tienen claros los parámetros a tener en cuenta para identificar el dolor se ha encontrado una considerable discrepancia, tanto en cómo aprecian el dolor los veterinarios, como en los métodos usados para controlarlo (Capner et al., 1999; Lascelles et al., 1999; Price et al., 2002; Hewson et al., 2007; Waran et al., 2010). Una gran proporción (40-60\%) de veterinarios clasifican su propia capacidad para reconocer el dolor y el conocimiento de la terapia analgésica en caballos solo moderada (Valverde y Gunkel, 2005). Debido a que sentir dolor es subjetivo, a menudo el médico veterinario se ve forzado a emitir un juicio sobre si un equino está o no experimentando dolor, con base en su práctica profesional (Schaafsma, 2009), así como en la interpretación de indicadores fisiológicos y conductuales (Price et al., 2002; Murrell y Johnson, 2006). Algunos autores han señalado que existe una correlación positiva entre la percepción humana del dolor y la de otros animales frente a determinadas condiciones dolorosas, y los niveles de empatía del hombre hacia otras especies animales (Ellingsen et al., 2010; Kielland et al., 2010).

Debido a que la identificación y cuantificación del dolor depende de la habilidad del observador como se mencionó anteriormente para detectar cambios fisiológicos y comportamentales relacionados con el mismo es imperativo que el observador: (I). determine cuales parámetros fisiológicos y comportamentales pueden estar asociados con dolor (II). Sistematice las observaciones de estos hallazgos, (III). Especifique el grado de dolor relacionado con cierto tipo de comportamiento y (IV). Combine estas observaciones a un puntaje cuantitativo (Ashley et al., 2005). 
Para identificar o facilitar la interpretación de los estados de dolor se han desarrollado varios métodos de evaluación entre los que encontramos la medición de los parámetros fisiológicos, escalas de dolor, prueba de umbral nociceptivo, análisis en el tiempo y test de analgesia. En cuanto a la medición de los parámetros fisiológicos se ha encontrado que equinos con cólico u otras enfermedades severas a moderadas la frecuencia cardiaca y la frecuencia respiratoria y la presión sanguínea pueden verse afectadas por el dolor, pero también pueden verse afectadas por endotoxemia, compromiso cardiovascular y/o deshidratación, además que en condiciones clínicas o de hospitalización estos parámetros pueden verse afectados por el tratamiento que está recibiendo el paciente, es decir que se debe tener total cuidado con la interpretación de estos hallazgos y su relación con el dolor (Pader et al., 2011). Por otra parte el acceso sistemático del dolor mediante escalas o sistemas de puntajes validados y definidos ayuda al reconocimiento y tratamiento de condiciones dolorosas que afectan a los equinos, ya que en estos se agrupa información de parámetros fisiológicos y comportamentales brindando una mejor correlación entre los observadores un récord más confiable sobre la severidad del dolor sobre el tiempo y mayores probabilidades de instaurar una terapéutica correcta (Dutton et al., 2009; Wagner, 2010). Usando el modelo de anfotericina B, Bussieres et al., (2008) desarrolló y validó una escala de dolor compuesta que evalúa el dolor de tipo ortopédico. Esta escala incluye variables de comportamiento interactivo, (respuesta a la palpación del área lesionada) observacional (postura) y fisiológico (Tabla 2). De las variables incluidas en esta escala, la respuesta a la palpación del área lesionada y la postura fueron los indicadores más sensibles y específicos del dolor asociado con sinovitis severa, de igual manera el modelo de sinovitis inducida por LPS ha sido utilizado para el estudio de la eficacia analgésica para el tratamiento del dolor articular encontrando buenos resultados terapéuticos con AINEs orales (de Grauw et al., 2009; van Loon et al., 2014), infusiones continuas de opioides, morfina epidural (Freitas et al., 2011; van Loon et al., 2012) y bupremorfina (Freitas et al., 2011). Esta escala muestra buena correlación entre los observadores, pero sensibilidad limitada para detectar el dolor moderado. (Lindegaard et al., 2010). Por otra parte, la escala de dolor compuesta desarrollada 
por Dalla Costa et al., (2014) tiene buena aplicabilidad para la evaluación de dolor post quirúrgico en equinos, principalmente después de procedimientos de castración, esta escala se basa en 6 parámetros faciales (posición de las orejas, tensión orbital, tensión de la boca, tensión de los músculos masticatorios, apertura de las narinas y aplanamiento del perfil (Tabla 3). Se encontró que esta escala de dolor de gestos en equinos tiene una buena correlación para la determinación de dolor post-castración además de esto permite la determinación de dolor moderado. Ashley et al., (2005) y Sutton et al., (2013) enfocaron su trabajo en el desarrollo de dos escalas para determinar el grado de dolor durante procesos de cólico agudo (EAAPS-1 y -2) (Tabla 4 y 5). Estas escalas multivariables están basadas principalmente en componentes de comportamiento observacional excluyendo la evaluación de parámetros fisiológicos, sin embargo la correlación entre observadores que utilizaron las escalas EAAPS-1 y -2 fue excelente (coeficiente de correlación de 0.8 y 0.76 respectivamente), indicando que estas pueden ser usadas como una herramienta objetiva útil para determinar el dolor abdominal agudo en manos de diferentes observadores, permitiendo a los clínicos tomar decisiones médicas, quirúrgicas o de eutanasia (Sutton et al., 2013). En la clínica equina otros de los principales procedimientos que se realizan a diario son las cirugías abdominales, para la determinación y evaluación del dolor post-quirúrgico Pritchett et al., (2003) desarrolló una escala numérica usando indicadores comportamentales y fisiológicos, con el fin de monitorear la eficacia analgésica. Haciendo uso del análisis en el tiempo en segmentos de una hora para cuantificar el comportamiento, Pritchett et al., (2003) encontró que, los equinos con procedimientos postquirúrgicos gastaban más tiempo expresando comportamientos de dolor y menos tiempo realizando locomoción voluntaria.

La escala numérica desarrollada por Pritchett et al., (2003) (Tabla 6), asigna un Puntaje numérico a cada categoría de comportamiento el cual está basado en la descripción de cada uno de ellos. Por otra parte, Sellon et al., (2004) utilizó esta escala numérica para medir la respuesta analgésica del butorfanol en procesos postquirúrgicos de coeliotomias, encontrando un efecto significativo al tratamiento y una alta sensibilidad para determinar comportamientos de dolor en estos pacientes, 
sin embargo, el modelo de escala desarrollado por Pritchett et al., (2003), no verifica la correlación entre los observadores. La prueba de umbral nociceptivo, el análisis en el tiempo y el test de analgesia son otro tipo de herramientas que se han venido utilizando para identificar el dolor en equinos sin embargo, requiere de equipos especializados y mayor conocimiento en el área para realizar un análisis correcto de dichos resultados. Por esto se considera que en la actualidad unos de los mejores métodos existentes para identificar y medir la efectividad de la terapéutica son mediante la aplicación de escalas de dolor, para condiciones específicas de cada paciente.

En cuanto a la terapéutica encontramos que existen varios grupos de fármacos disponibles en el mercado para el tratamiento del dolor en equinos, sin embargo, estos fármacos presentan un gran número de efectos adversos que generan sobre el paciente lo que hace considerar si es imperativo o no su uso.

La analgesia polimodal, es un nuevo concepto en el manejo del dolor esta se basa en la asociación de fármacos de diferentes grupos que usados de manera simultánea y correcta permiten lograr un efecto seguro y eficaz. A través de dichas asociaciones, se alcanza un sinergismo analgésico (mayor eficacia), con reducción de los efectos adversos generados por los fármacos (mayor seguridad) en su aplicación, no solo se concibe la asociación farmacológica sino la utilización de diferentes vías de administración de manera simultánea, todo ello para obtener los mayores beneficios de cada fármaco utilizado (Flecknell, 2008; Matthews y Caroll 2007; Reyes et al., 2004). Se considera que el mejor efecto analgésico bajo la concepción de la analgesia polimodal, se logra al asociar, como se ha mencionado, fármacos con diferentes mecanismos de acción, pero principalmente cuando se administran simultáneamente fármacos que ejercen la acción analgésica a nivel central con otros que lo hacen a nivel periférico (Taylor, 2007). Los fármacos antiinflamatorios utilizados en equinos incluyen corticoides y AINEs, estos son usualmente la primera línea de elección en el manejo del dolor relacionado con condiciones de tipo inflamatorio (sinovitis y osteoartritis) (Owens et al., 1996). Generalmente los AINEs son los medicamentos más comúnmente utilizados en 
equinos debido a su disponibilidad y bajo costo (Goodrich y Nixon, 2006). La fenilbutazona (4.4 mg/kg IV q 24 horas durante 4 días) y el flunixin meglumine (1.1 $\mathrm{mg} / \mathrm{kg}$ IV q 24 horas durante 4 días) han sido estudiados con eficacia para el tratamiento del síndrome navicular (Erkert et al., 2005). Por su parte el firocoxib un AINEs selectivo de la COX-2 utilizado IV a dosis de $0.09 \mathrm{mg} / \mathrm{kg} \mathrm{q} 24 \mathrm{~h}$, demostró ser efectivo en el tratamiento de la osteoartritis (Orsini et al., 2012). El meloxicam es utilizado comúnmente en la práctica equina en otros países, su eficacia en equinos ha sido establecida para el manejo de dolor post operatorio ortopédico e inflamación. De Grauw et al., 2009 evaluó experimentalmente el meloxicam (0.6 $\mathrm{mg} / \mathrm{kg}$ vía oral q 24 horas durante 7 días) este mostró ser efectivo para el tratamiento de sinovitis agudas, produciendo una disminución de los biomarcadores inflamatorios en el líquido sinovial y reduciendo significativa de la cojera. Otro AINEs utilizado con menor frecuencia es el diclofenaco, actualmente el único producto veterinario aprobado y disponible en el mercado es en presentación liposomal tópica (73 mg q $12 \mathrm{~h}$ ), indicada para el control de dolor asociado con inflamación principalmente osteoartritis (Caldwell et al., 2004). Otro de los usos rutinarios de los AINEs en la clínica equina es durante el dolor abdominal en este se utiliza para reducir los efectos de la endotoxemia (flunixin meglumine $0,2 \mathrm{mg} / \mathrm{kg} \mathrm{IV}$ ) y el dolor visceral (flunixin meglumine $1.1 \mathrm{mg} / \mathrm{kg}$ IV q 12-24 horas (Baskett et al., 1997; Shuster et al., 1997). Otro grupo de fármacos importante para el manejo del dolor son los opioides, la morfina es el Agonista- $\mu$ con el cual se comparan los demás opioides (Combie et al., 1981; Combie et al., 1983). Tradicionalmente se conoce que los Agonistas- $\mu$ completos proveen el efecto analgésico más potente, sin embargo el butorfanol (0.01-0.22 mg/kg IV, IM, SC) es reportado por ser igual o más efectivo (con menos potencial de efectos adversos) que los Agonistas- $\mu$ en el tratamiento del dolor gastrointestinal en equinos Sellon et al., (2004). El uso de opioides (morfina 0.05-0.2 mg/kg IV, IM) con agonistas-a2 (0.2-1.1 mg/kg IV, IM) produce un potente efecto sedativo el cual puede ser útil en animales refractarios a la analgesia o cuando se desean realizar procedimientos quirúrgicos (Robertson et al., 2005). El tramadol es otro opioide que se ha usado principalmente para el tratamiento del dolor óseo o articular, la administración epidural de tramadol (1.0 
$\mathrm{mg} / \mathrm{kg}$ ) resultó en una disminución significativa de la nocicepción luego de 3 horas posteriores a su aplicación (Natalini y Robinson, 2000). Los anestésicos locales (lidocaína, mepivacaina, bupivacaina, ropivacaina) son frecuentemente utilizados solos o en combinación con otros fármacos analgésicos, estos pueden ser administrados por vía tópica, regional (peri neural, epidural) o sistémicos (Doherty y Frazier, 1998). Cuando son utilizados estos producen analgesia, disminuyendo los requerimientos de anestésicos inhalados, y potenciando la acción analgésica de los opioides y agonistas- $\alpha 2$ adrenérgicos. El uso intra articular de los anestésicos locales para el tratamiento del dolor articular, diagnóstico de cojeras y cirugías articulares es común en la práctica equina, sin embargo, en un estudio in vitro en equinos se observó que el uso de bupivacaina (0.02-0.06 mg/kg IA), lidocaína (0.2$0.35 \mathrm{mg} / \mathrm{kg})$ y mepivacaina $(0.16-0.2 \mathrm{mg} / \mathrm{kg} \mathrm{IA})$ se ha asociado con condrotoxicidad, sin embargo, este estudio mostró que el menor efecto de toxicidad se logra con la mepivacaina (Park et al., 2011). La lidocaína ha sido igualmente utilizada para el tratamiento de afecciones gastrointestinales en un bolo inicial de $1.3 \mathrm{mg} / \mathrm{kg}$ seguido de infusiones constantes de $50 \mathrm{mg} / \mathrm{kg} / \mathrm{min}$ mostrando un buen efecto en promover la motilidad y reduciendo efectivamente el dolor abdominal (Feary et al., 2005). Por su parte los agonistas $\alpha 2$ son analgésicos viscerales potentes, esta propiedad es crucial para el manejo del cólico moderado a severo (Jochle et al., 1989). Los agonistas a2 pueden ser administrados en bolos, dosis, infusión constante o como los opioides también pueden ser administrados por vía intra articular, peri neural y epidural (Di Salvo et al., 2014). Uno de los agonistas $\alpha 2$ mayormente utilizados es la xilacina (IV, IM 0.2-1.1 mg/kg; Epidural 0.17-0.22 mg/kg; IV CRI 0.016-0.69 $\mathrm{mg} / \mathrm{kg} / \mathrm{h}$ ), debido al corto efecto sedativo de la xilacina cuando se asocia con el butorfanol (IV, IM, SC 0.01-0.22 $\mathrm{mg} / \mathrm{kg}$ ) actúan como una excelente opción para evaluación y tratamiento del dolor abdominal agudo en equinos (Merritt et al., 1998).

\section{CONCLUSIONES Y RECOMENDACIONES}

Enfocar la evaluación del dolor es importante para asegurar el bienestar de los pacientes equinos, para desarrollar estas habilidades es de gran importancia para los veterinarios conocer los tipos de dolor, los aspectos fisiopatológicos, los métodos 
de identificación y las alternativas terapéuticas que ofrece el mercado para el tratamiento del dolor en equinos. Las escalas de dolor se han convertido en una herramienta diagnostica importante del dolor en caballos, estas permiten identificar el dolor y evaluar la respuesta a la analgesia, la experiencia y el tiempo de observación son factores que influye en la correlación de la respuesta de los observadores, sin embargo, existen escalas diseñadas que pueden ser utilizadas para patologías específicas que aquejan a los equinos las cuales han demostrado buenos resultados entre varios observadores. El manejo farmacológico del dolor en animales, ha mostrado valiosos avances relacionados con la introducción de nuevos conceptos terapéuticos para la utilización de analgésicos ya existentes, aunque no se evidencia la generación de nuevos grupos farmacológicos, la combinación de los mismos potencia el efecto de estos disminuyendo la cantidad de dosis requerida y a su vez los efectos adversos que su uso genera. la educación continua y la investigación en el manejo del dolor ayudarán a mejorar los métodos para la evaluación objetiva y reproducible del dolor. Apoyar el desarrollo de nuevos fármacos analgésicos y más técnicas analgésicas en consecuencia tendrá un efecto positivo sobre el bienestar animal.

\section{REFERENCIAS BIBLIOGRÁFICAS}

1. Alexander SL., Irvine CH. 2000. The effect of the alpha-2-adrenergic agonist, clonidine, on secretion patterns and rates of adrenocorticotropic hormone and its secretagogues in the horse. J Neuroendocrinol. 9: 874-880.

2. Ashley, FH., Waterman-Person, AE, Whay, HR. 2005. Behavioural assessment of pain in horses and donkeys: application to clinical practice and future studies. Equine Veterinary Journal, 37: 565-575.

3. Bailey, SR y Elliot, J. 2007. The corticosteroid laminitis story: 2. Science of if, when and how. Equine Vet J, 39: 7-11.

4. Baskett, A., Barton, MH., Norton, N. 1997. Effect of pentoxifylline, flunixin meglumine and their combination on a model of endotoxemia in horses. Am J Vet Res. 58: 1292-1299.

5. Bateson, P. 1991. Assessment of pain in animals. Anim. Behav. 42: 827-839.

6. Blackshaw, LA, Gabhart, GF. 2002. The pharmacology of gastrointestinal nociceptive pathways. Curr Opin Pharmacol, 2: 642.

7. Bennett, RC., Steffey, EP. 2002. Use of opioids for pain and anesthetic management in horses. Vet Clin North Am Equine Pract, 18: 47-110.

8. Bisgaard, T., Kehlet, H., Rosenberg, J. 2001 Pain and convalescence after laparoscopic cholecystectomy. Eur. J. Surg.167: 184. 
9. Bueno, L., Fioramonti, J, Delvaux, et al. 1997. Mediators and pharmacology of visceral sensitivity: from basic to clinical investigations. Gastroenterology, 112: 1714.

10. Bussieres, G., Jacques, C., Lainay, O., Beauchamp, G., Leblond, A., Cadore, JL., Desmaizieres, LM., Cuvelliez, SG., Troncy, E. 2008. Development of a composite orthopaedic pain scale in horses. Research in Veterinary Science, 85: 294-306.

11. Büttner, W., Finke, W. 2000. Analysis of behavioural and physiological parameters for the assessment of postoperative analgesic demand in newborns, infants and young children: A comprehensive report on seven consecutive studies. Pediatric Anesthesia, 10: 303-318.

12. Caldwell, FJ., Mueller, E., Lynn, RC. 2004. Effect of topical application of diclofenaco liposomal suspension on experimentally induced subcutaneous inflammation in horses. Am J Vet Res, 65: 271-276.

13. Capner CA., Lascelles, BD., Waterman-Pearson AE. 1999. Current British veterinary attitudes to perioperative analgesia for dogs. Veterinary Record, 145: 95-99.

14. Combie, J., Shults, T., Nugent, EC, et al. 1981. Pharmacology of narcotic analgesics in the horse: selective blockade of narcotic-induced locomotor activity. Am J Vet Res, 42: 716.

15. Combie, J., Blake, JW., Ramey, BE, et al. 1981. Pharmacology of narcotic analgesics in the horse: quantitative detection of morphine in equine blood and urine and logit-log transformations of this data. Am J Vet Res, 42: 1523.

16. Combie, J., Nugent, TE, Tobin, T. 1983. Pharmacokinetics and protein binding of morphine in horses. Am J Vet Res, 44: 870.

17. Cook, VL., Meyer, CT., Campbell, NB, et al. 2009. Effect of firocoxib or flunixin meglumine on recovery of ischemic-injured equine jejunum. Am J Vet Res, 70: 9921000.

18. Dalla, E., Minero, M., Lebelt, D., Stucke, D., Canali, E., Leach, MC. 2014. Development of the horse grimace scale (HGS) as a pain assessment tool in horses undergoing routine castration. PLoS ONE 9: e92281.

19. Daunt DA., Steffey EP. 2008. Alpha-2 adrenergic agonists as analgesic in horses. Vet Clin North Am Equine Pract, 18: 39-46.

20. de Grauw, JC., van de Lest, CH., Brama, PA., Rambags, BP., vanWeeren, PR. 2009. In vivo effects of meloxicam on inflammatory mediators, MMP activity and cartilage biomarkers in equine joints with acute synovitis. Equine Veterinary Journal, 41: 693-699.

21. Di Salvo, A., Della Rocca, G., Bazzica, C. 2014. A pharmacokinetic/ clinical approach to postulate a local action of intra-articular xylazine administration in the horse: a preliminary study. J Vet Pharmacol Ther, 37: 464.

22. Dobromylskyj, P., Flecknell, PA., Lascelles, BD., Livingston, A., Taylor, P., Waterman-Pearson, A. 2000. Pain assessment. In: Flecknell, P.A., WatermanPearson, A. (Eds.), Pain Management in Animals, pp.53-79.

23. Doherty, TJ, Frazier, DL. 1998. Effect of intravenous lidocaine on halothane minimum alveolar concentration in ponies. Equine Vet J, 30: 300.

24. Dujardin, CL, van Loon, JP. 2011. Pain recognition and treatment in the horse: $A$ survey of equine veterinarians in The Netherlands and Belgium. Tijdschrift Voor Diergeneeskunde, 136: 715-724.

25. Dutton, DW, Lashnits, KJ, Wegner, K. 2009. Managing severe hoof pain in a horse using multimodal analgesia and a modified composite pain score. Equine Veterinary Education, 21: 37-43. 
26. Dzikiti, TB., Hellebrekers, LJ., van Dijk, P. 2003. Effects of intravenous lidocaine on isoflurane concentration, physiological parameters, metabolic parameters and stress-related hormones in horses undergoing surgery. Journal of Veterinary Medicine. A, Physiology, Pathology, Clinical Medicine, 50: 190-195.

27. Elfenbein, JR., Sanchez, LC., Robertson, SA., et al. 2009. Effect of detomidine on visceral and somatic nociception and duodenal motility in conscious adult horses. Vet Anaesth Analg, 36: 162-172.

28. Ellingsen, K., Zanella, AJ., Bjerkås, E., Indrebo, A. 2010. The relationship between empathy, perception of Pain and attitudes toward pets among Norwegian dog owners. Anthrozoös, 23: 231-243.

29. Erkert, RS., MacAllister, CG., Payton, ME. 2005. Use of force plate analysis to compare the analgesic effects of intravenous administration of phenylbutazone and flunixin meglumine in horses with navicular syndrome. Am J Vet Res, 66: 284-288.

30. Feary, DJ., Mama, KR., Wagner, AE. 2005. Influence of general anesthesia on pharmacokinetics of intravenous lidocaine infusion in horses. Am J Vet Res, 66: 574-580.

31. Freeman, SL., Curtis, L. 2015. Developing best practice guidelines on equine colic. Veterinary Record, 10: 38-40.

32. Freitas, GC., Carregaro, AB., Gehrcke, MI., De La Corte, FD., Lara, VM., Pozzobon, R., Brass, KE. 2011. Epidural analgesia with morphine or buprenorphine in ponies with lipopolysaccharide (LPS)-induced carpal synovitis. Canadian Journal of Veterinary Research, 75: 141-146.

33. Giamberardino, M 2005. Visceral pain. International association for the study of pain, Clinical Updates, 8: 1-6.

34. Gleerup, KB, Lindergaard, C. 2016. Recognition and quantification of pain in horses: A tutorial review. Equine Veterinary Education, 28: 47-57.

35. Gleerup, KB., Forkman, B., Lindegaard, C, Andersen, PH. 2014. An equine pain face. Vet. Anaesth Analg, 42, 103-114.

36. Goodrich, LR, Nixon, AL. 2006. Medical treatment of osteoarthritis in the horse-a review. Vet J, 171: 51-69.

37. Harding, EJ., Paul, ES., Mendl, M. 2004. Animal behaviour: cognitive bias and affective state. Nature, 427: 312.

38. Harkins, JD., Stanley., Mundy, GD, et al. 1995. A review of the pharmacology, pharmacokinetics, and regulatory control in the US of local anaesthetics in the horse. J Vet Pharmacol Ther, 18: 397.

39. Hewson, CJ., Dohoo, IR., Lemke, KA., Barkema, HW. 2007. Canadian veterinarians' use of analgesics in cattle, pigs, and horses in 2004 and 2005. Canadian Veterinary Journal. 48: 155-164.

40. Hielm-bjorkman, AK., Kapatkin, AS., Rita, HJ. 2011. Reliability and validity of a visual analogue scale used by owners to measure chronic pain attributable to osteoarthritis in their dogs. Am. J. Vet. 72: 601-607.

41. Hunter, JO. 2009. Do horses suffer from irritable bowel syndrome? Equine Vet $J$. 41: 836-840.

42. Jochle, W., Moore, JN., Brown J. 1989. Comparison of detomidine, butorphanol, flunixin meglumine and xylazine in clinical cases of equine colic. Equine Vet J Suppl. 7: 111.

43. Kielland, C., Skjerve, E., Zanella, AJ. 2009. Attitudes of veterinary students to pain in cattle. Veterinary Record. 165: 254-258. 
44. Kalpravidh, M., Lumb, WV., Wright, M., et al. 1984. Effects of butorphanol, flunixin, levor-phanol, morphine, and xylazine in ponies. Am J Vet Res, 45: 217-223.

45. Kester, WO. 1991. Definition and Classification of Lameness. Guide for Veterinary Service and Judging of Equestrian Events. American Association of Equine Practitioners (AAEP), 19.

46. Lang, PJ., Davis, M., Öhman, A. 2000. Fear and anxiety: animal models and human cognitive psychophysiology. Affect. Disord, 61: 137-159.

47. Lascelles, BD., Capner, CA., Waterman-Pearson, AE. 1999. Current British veterinary attitudes to perioperative analgesia for cats and small mammals. Veterinary Record. 145: 601-604.

48. Leigh, JP., Seavey, W, Leistikow, B, 2001. Estimating the costs of job related arthritis. J Rheumatol, 28: 1647-1654.

49. Lindegaard, C., Thomsen, MH., Larsen, S., Andersen, PH. 2010. Analgesic efficacy of intra-articular morphine in experimentally induced radiocarpal synovitis in horses. Veterinary Anaesthesia and Analgesia, 37: 171-185.

50. Love, EJ., Taylor, PM., Clark, C., Whay, HR., Murrell, J. 2009. Analgesic effect of butorphanol in ponies following castration. Equine Veterinary Journal, 41: 552-556.

51. Love, EJ., Taylor, PM., Whay, HR., Murrell, J, 2013. Postcastration analgesia in ponies using buprenorphine hydrochloride. Veterinary Record, 172: 635.

52. MacAllister, CG., Morgan, SJ., Borne, AT, Pollet RA. 1993. Comparison of adverse effects of phenylbutazone, flunixin meglumine, and ketoprofen in horses. J Am Vet Med As-soc, 202: 71-77.

53. Mair, T., Divers, T, Ducharme N. 2002. Etiology, risk factors, and pathophysiology of colic. Manual of equine gastroenterology, pp. 101-106.

54. Matthews, N, Carroll, GL. 2007. Review of Equine Analgesics and Pain Management. AAEP Proceedings, 53: 240-245.

55. Merritt, AM., Burrow, JA., Hartless, CS. 1998. Effect of xylazine, detomidine, and a combination of xylazine and butorphanol on equine duodenal motility. Am J Vet Res, 59: 619-623.

56. Molony, V., Kent, J.E. 1997. Assessment of acute pain in farm animals using behavioural and physiological measurements. Journal of Animal Science, 75: 266272.

57. Muir, WW., Skarda, RT, Sheehan, W. 1979. Hemodynamic and respiratory effects of xylazine-morphine sulfate in horses. Am J Vet Res, 40: 1417.

58. Muir, W, Woolf, CJ. 2001. Mechanisms of pain and their therapeutic implications. $J$ Am Vet Med Assoc, 219: 1346.

59. Muir, W. 2010. Mechanisms and management in horses. Vet Clin Equine, 26: 467480.

60. Muir, W. 2016. Its diagnosis and management in the rehabilitation of horses. Vet Clin Equine, 32: 13-29.

61. Murrell, JC., Johnson, CB. 2006. Neurophysiological techniques to assess pain in animals. Journal of Veterinary Pharmacology and Therapeutics. 29: 325-335.

62. Natalini, CC., Driessen, B. 2007. Epidural and spinal anesthesia and analgesia in the equine. Clinical Techniques in Equine Practice, 6: 145-153.

63. Natalini, CC., Robinson, EP. 2000. Evaluation of the analgesic effects of epidurally administered morphine, alfentanil, butorphanol, tramadol, and U50488H in horses. Am J Vet Res. 6: 1579-1586. 
64. Obel, N. 1948. Studies on the histopathology of acute laminitis. PhD Dissertation. Swedish University of Agricultural Sciences. Almqvist and Wiksells, Uppsala, Sweden.

65. Orsini, JA., Ryan, WG., Carithers, DS. 2012. Evaluation of oral administration of firocoxib for the management of musculoskeletal pain and lameness associated with osteoarthritis in horses. Am J Vet Res, 73: 664-671.

66. Owens, JG., Kamerling, SG., Stanton, SR, Keowen, ML. 1995. Effects of ketoprofen and phenylbutazone on chronic hoof pain and lameness in the horse. Equine vet. J, 27: 296-300.

67. Owens JG., Kamerling, SG., Stanton, SR. 1996. Effects of pretreatment with ketoprofen and phenylbutazone on experimentally induced synovitis in horses. $A m$ $J$ Vet Res, 57: 866.

68. Pader, K., Freeman, LJ., Constable, PD., Wu, CC., Snyder, PW., Lescun, TB. 2011. Comparison of transvaginal natural orifice transluminal endoscopic surgery (NOTES_) and laparoscopy for elective bilateral ovariectomy in standing mares. Vet. Surg, 40: 998-1008.

69. Park, J., Sutradhar, BC., Hong G. 2011. Comparison of the cytotoxic effects of bupivacaine, lidocaine, and mepivacaine in equine articular chondrocytes. Vet Anaesth Analg, 38: 127.

70. Price, J., Marques, JM., Welsh, EM, Waran, NK. 2002. Pilot epidemiological study of attitudes towards pain in horses. Vet Rec. 151: 570-575.

71. Price, J., Catriona, S., Welsh, EM, Waran, NK. 2003. Preliminary evaluation of a behaviour-based system for assessment of post-operative pain in horses following arthroscopic surgery. Veterinary Anaesthesia and Analgesia, 30: 132.

72. Pozzi, A., Muir, W., Traverso, F. 2006. Prevention of central sensitization and pain by N-methyl- D-aspartate receptor antagonists. J Am Vet Med Assoc, 1: 53-60.

73. Pritchett, LC., Ulibarri, C., Roberts, MC., Schneider, RK., Sellon, DC. 2003. Identification of potential physiological and behavioral indicators of postoperative pain in horses after exploratory celiotomy for colic. Applied Animal Behaviour Science, 80: 31-43.

74. Raekallio, M., Taylor, PM, Bennett, RC. 1997. Preliminary investigations of pain and analgesia assessment in horses administered phenylbutazone or placebo after arthroscopic surgery. Vet Surg, 26: 150-155.

75. Reyes, FA., De la Gala, GF., Garutti, I. 2004. Dolor postoperatorio: Analgesia multimodal. Patología del Aparato Locomotor, 2: 176-188.

76. Rezende, ML., Wagner, AE., Mama, KR, et al. 2011. Effects of intravenous administration of lidocaine on the minimum alveolar concentration of sevoflurane in horses. Am J Vet Res, 72, 446.

77. Robertson., S.A. 2002. What is pain? Journal American Veterinary Medical Association, 221: 202-205.

78. Robertson, SA., Sanchez, CL. 2010. Treatment of visceral pain in horses. Vet Clin Equine, 26: 603-617.

79. Robertson, SA., Sanchez, LC y Merritt, AM, et al. 2005. Effect of systemic lidocaine on visceral and somatic nociception in conscious horses. Equine Vet J, 37: 122127.

80. Salazar, V. 2012. Analgesia multimodal en el paciente equino. I congreso solidário de clinica equina, pp. 59-61.

81. Schaafsma, MK. 2009. Assessment of pain in horses after surgical castration. Composition of a pain scale [tesis doctoral, en línea]. Países Bajos: Faculty of 
Veterinary Medicine, Utrecht University. [Consultado: 08 Sept. 2015]. Disponible En: http://dspace.library.uu.nl/handle/1874/33617

82. Sellon, DC., Monroe, VL., Roberts, MC et al. 2001. Pharmacokinetics and adverse effects of butorphanol administered by single intravenous injection or continuous intra- venous infusion in horses. Am J Vet Res, 62: 183-189.

83. Sellon, DC., Roberts, MC., Blikslager, AT., Ulibarri, C., Papich, MG. 2004 Effects of continuous rate intravenous infusion of butorphanol on physiologic and outcome variables in horses after celiotomy. J. Vet. Intern. Med, 18: 555-563.

84. Shuster, R., Traub-Dargatz, J., Baxter G, 1997. Survey of diplomates of the American College of Veterinary Internal Medicine and the American College of Veterinary Surgeons regarding clinical aspects and treatment of endotoxemia in horses. J Am Vet Med Assoc, 210: 87-92.

85. Sutton, GA., Dahan, R., Turner, D, Paltiel, O. 2013. A behaviour-based pain scale for horses with acute colic: Scale construction. The Veterinary Journal, 196: 394401.

86. Taylor, PM., Pascoe, PJ, Mama, KR. 2002. Diagnosing and treating pain in the horse - Where are we today?. Vet Clin North Am Equine Pract., 18: 1-19.

87. Taylor, MA. 2007. Control of pain in horses in field practice. J Vet Intern Med, 3: 302-307.

88. Valverde, A., Gunkel, C.I., 2005. Pain management in horses and farm animals. Journal of Veterinary Emergency and Critical Care, 15: 295-307.

89. Van Ioon, JP., Back, W, Hellebrekers, LJ. 2010. Application of a composite pain scale to objectively monitor horses with somatic and visceral pain under hospital conditions. Journal Equine Vet Sci, 30: 641-649.

90. Wagner, A. 2010. Effects of stress on pain in horses and incorporating pain scales for equine practice. Veterinary Clinics of North America Equine Practice Journal, 26: 481-492.

91. Waran N., Williams VM., Clarke N., Bridge IS. 2010. Recognition of pain and use of analgesia in horses by veterinarians in New Zealand. New Zealand Veterinary Journal. 58: 274-280.

92. White, Jp., Cibelli, M y Fidalgo, AR et al. 2010. Role of transient receptor potential and acid-sensing ion channels in peripheral inflammatory pain. Anesthesiology Journal, 112: 729- 741.

93. Viñuela-Fernández, I., Jones, E., Chase-topping, ME, Price, J. 2011. Comparison of subjective scoring systems used to evaluate equine laminitis. The Veterinary Journal, 188: 171-177. 